CARDIOVASCULAR MEDICINE

\title{
Impact of nicorandil in angina: subgroup analyses
}

IONA Study Group

Heart 2004;90:1427-1430. doi: 10.1136/hrt.2003.026310

\begin{abstract}
Aims: IONA (impact of nicorandil in angina) is a randomised, double blind, placebo controlled trial of nicorandil, with a target dose of $20 \mathrm{mg}$ twice daily. The consistency of benefits seen in subgroups is reported.

Methods: The primary composite end point of the study was coronary heart disease death, non-fatal myocardial infarction, or unplanned hospitalisation for cardiac chest pain. Subgroups were defined using baseline characteristics including, age, sex, histories of smoking, diabetes, hypertension, myocardial infarction, revascularisation, anginal status, anti-anginal treatment, other cardiovascular drugs, and an overall assessment of risk.

Results: A total of 5126 patients were randomised to receive nicorandil or identical placebo in addition to standard anti-anginal treatment. Overall, nicorandil reduced the incidence of the primary end point from $15.5 \%$ to $13.1 \%$ (hazard ratio (HR) $0.83,95 \%$ confidence interval $(\mathrm{Cl}) 0.72$ to $0.97 ; \mathrm{p}=0.014$ ). There was no evidence of significant heterogeneity of benefit across all subgroups studied. The absolute risk reduction was greatest and the numbers needed to treat to prevent one event was lowest in subjects at greatest risk.

Conclusions: The IONA study demonstrates a significant improvement in outcome by nicorandil treatment across a broad range of patients with stable angina.
\end{abstract}

Correspondence to:

Professor I Ford, Robertson

Centre for Biostatistics,

University of Glasgow,

Glasgow G12 8QQ, UK;

ian@stats.gla.ac.uk

Accepted 5 March 2004
A ngina pectoris is the most common manifestation of coronary heart disease, which is the major cause of death in Europe and North America. ${ }^{12}$ The symptoms and the morbidity related to angina lead to significant health care expenditure. ${ }^{3}$ Aspirin, angiotensin converting enzyme (ACE) inhibitors, and statins have been shown to reduce the risk of cardiovascular events in patients with coronary disease. ${ }^{4-8}$ Recently, the IONA (impact of nicorandil in angina) trial became the first study to demonstrate a similar effect with a specific anti-anginal agent. ${ }^{9}$ The properties of nicorandil have been described elsewhere. ${ }^{10-20}$

The objective of the current analysis was to investigate whether or not the results of IONA were consistent across subgroups.

\section{METHODS}

The design of IONA has been reported elsewhere. ${ }^{21}$ Briefly, patients with a history of angina were recruited from hospital and primary care centres in the UK. Standard background anti-anginal treatment was to be optimal therapy as judged by the investigator for the individual patient. Patients were randomised to a double blind comparison of nicorandil ( $10 \mathrm{mg}$ twice daily for two weeks and $20 \mathrm{mg}$ twice daily thereafter) and placebo. High risk men over 45 years, or women over 55 years, were recruited. Follow up was for an average of 1.6 years (range $1-3$ years). The primary end point of the study was the composite of coronary heart disease death, non-fatal myocardial infarction, or unplanned hospitalisation for cardiac chest pain. ${ }^{21}$

Risk factor subgroups were defined as history of diabetes, hypertension, myocardial infarction, coronary revascularisation (all yes/no); current smoking status (yes/no), age $(<65$, 65-70 and $>70$ years), sex; background medication with $\beta$ blockers, calcium channel blockers, long acting nitrates, diuretics, ACE inhibitors, aspirin, statins (all yes/no); numbers of anti-anginal medications being taken (one, two, or three drugs from $\beta$ blockers, calcium channel blockers, or long acting nitrates). Baseline anginal severity was assessed using the Canadian Cardiovascular Society
(CCS) functional status questionnaire with three groupings (I, II, and III + IV). A subgroup analysis was conducted using the 18 month risk of a primary end point calculated from a model which had been fitted to the placebo group using age, systolic blood pressure, heart rate, body mass index, sex, CCS functional status, smoking status, and histories of hypertension, diabetes, left ventricular hypertrophy, and left ventricular function. All analyses were conducted on the intentionto-treat principle and were based on the log rank test and Cox regression analysis as appropriate, including a test of homogeneity of the treatment effect across levels of subgroups.

Patients provided written informed consent and the study complied with the Declaration of Helsinki and was approved by the multi-centre research ethics committees of the UK.

\section{RESULTS}

In the trial, 2565 patients were randomised to nicorandil and 2561 to placebo. Overall, nicorandil reduced the incidence of the primary end point from $15.5 \%$ to $13.1 \%$ (hazard ratio (HR) 0.83 , 95\% confidence interval (CI) 0.72 to 0.97; $\mathrm{p}=0.014) .{ }^{8}$ The event rates and risk reduction were similar in patients recruited in general practice and in hospitals (data not shown). The results of the subgroup analyses for the baseline traditional risk factors are given in fig 1, for baseline medication in fig 2, and for baseline risk in fig 3. Although evidence of benefit is not established statistically in all subgroups, all point estimates of hazard ratios lie on, or to the left, of unity and none of the tests for interaction achieve significance. There is consistency of risk reduction across the tertiles of baseline risk with the number needed to treat (NNT) for 18 months in the three subgroups being respectively 28, 46 and 63 for the high, medium, and low risk groups.

Abbreviations: $A C E$, angiotensin converting enzyme; CCS, Canadian Cardiovascular Society; IONA, impact of nicorandil in angina; NNT, number needed to treat 


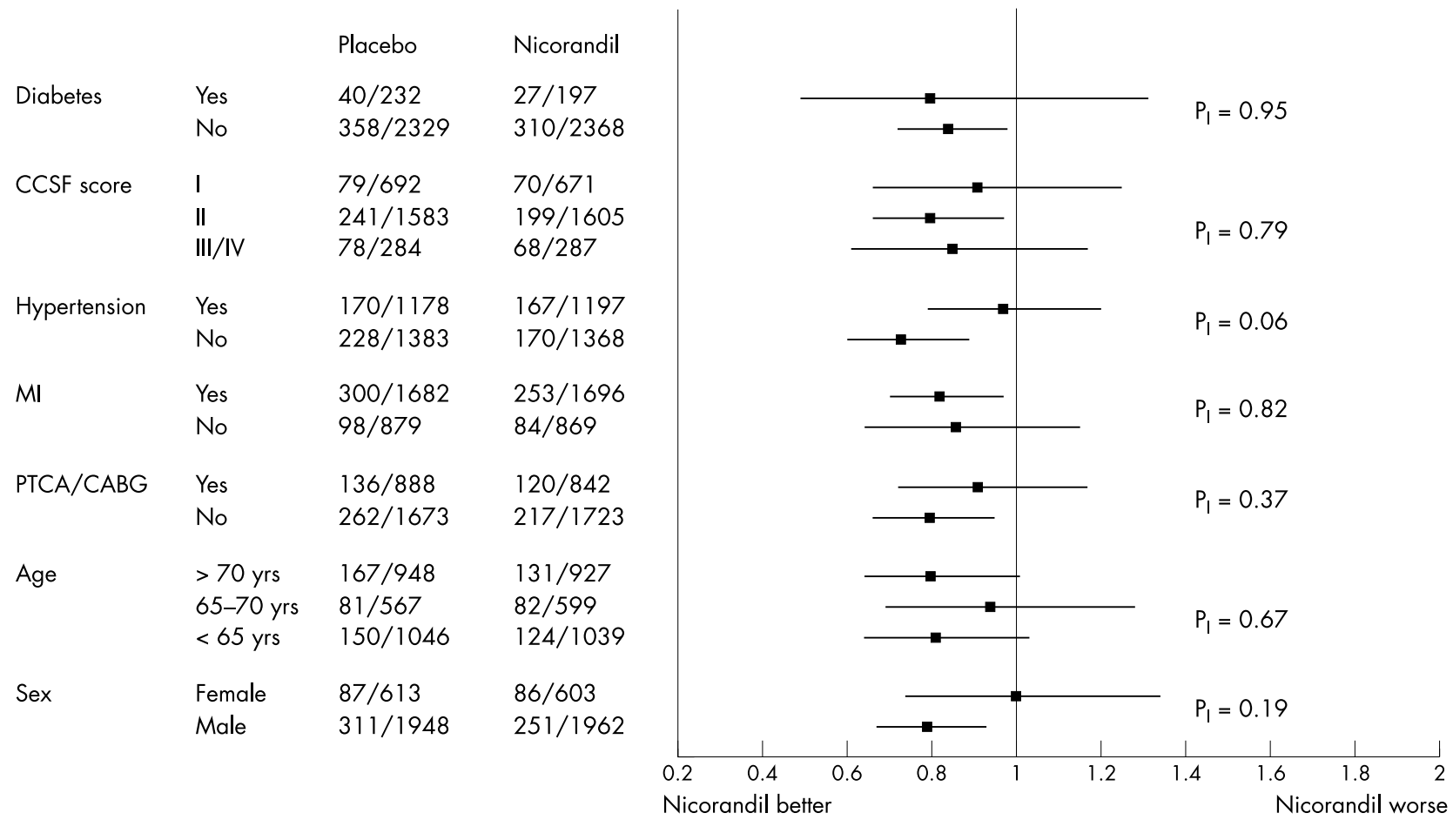

Hazard ratio

Figure 1 Estimated hazard ratios and $95 \% \mathrm{Cl}$ for subgroups defined by traditional risk factors analysed on the basis of the primary end point; $\mathrm{p}$ values are for tests of homogeneity of treatment effect. The numbers tabulated are the numbers of events/subjects for the placebo and nicorandil groups.

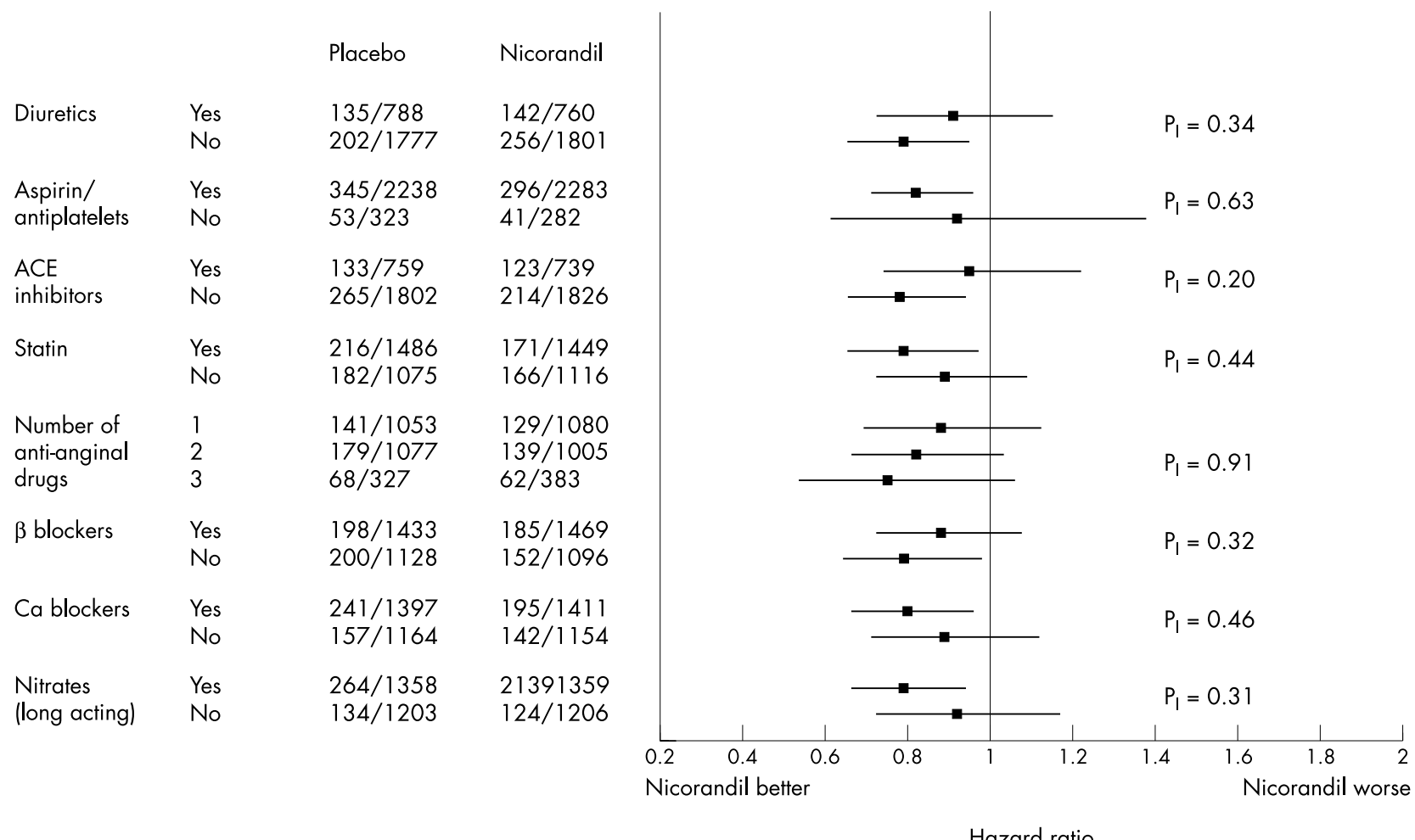

Figure 2 Estimated hazard ratios and $95 \% \mathrm{Cl}$ for subgroups defined by background medication risk factors analysed on the basis of the primary end point; $p$ values are for tests of homogeneity of therapy effect. The numbers tabulated are the numbers of (subjects with events)/(total number of subjects) for the placebo and nicorandil groups. 


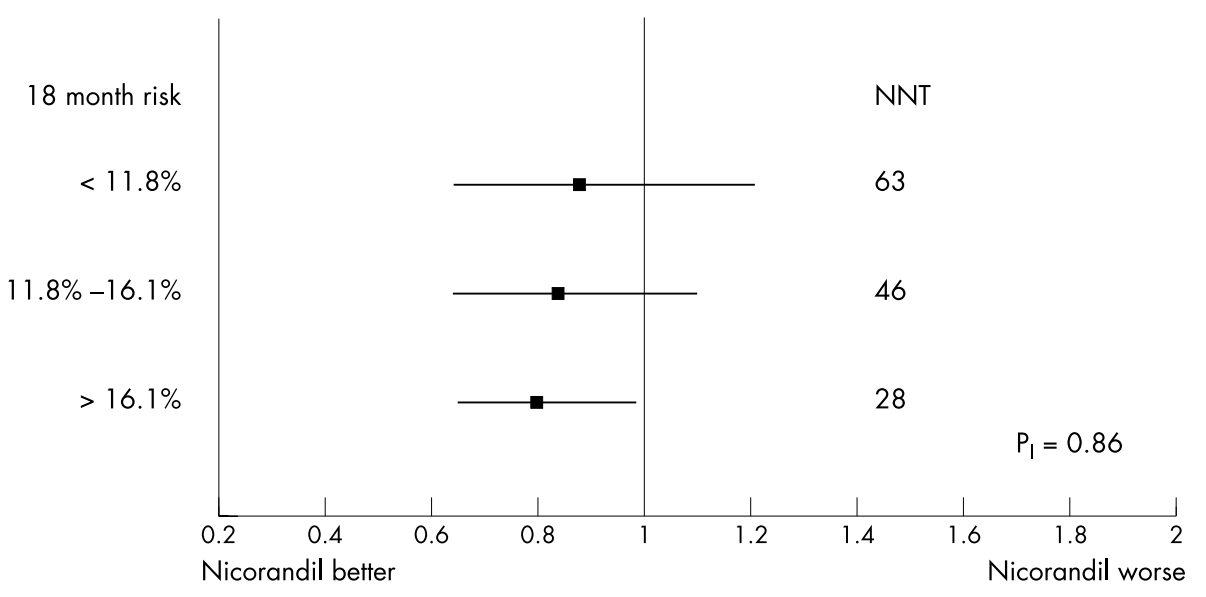

Figure 3 Hazard ratios for treatment effect and $95 \% \mathrm{Cl}$ based on the primary end point for subgroups defined by tertiles of baseline 18 month risk of a primary end point; $p$ value is for test of homogeneity of magnitude treatment across subgroups. NNT denotes the number of subjects that have to be initiated on treatment to prevent one subject having a primary end point.

Hazard ratio

\section{DISCUSSION}

The subgroup analyses provide no significant evidence of any qualitative or quantitative interactions between nicorandil treatment benefit and subgroup status. The effect of nicorandil on top of nitrates adds support to the argument that the mechanism of benefit is other than the nitrate properties of nicorandil and plausibly caused by its potassium channel opening activity. The hazard ratio for treatment benefit was at least as great in subjects receiving three antianginal medications as it was in those receiving one or two medications. A similarly consistent result was achieved across the categories of the CCS functional status questionnaire. Subjects taking higher numbers of anti-anginal medications, or with higher CCS functional status scores, were at particularly high risk. A reduction in relative risk that is independent of baseline risk suggests that treatment of those at highest risk will provide the greatest absolute risk reduction and possibly be the most cost effective. Our results suggest that treating subjects in the highest third of risk will prevent more than twice the number of events compared to the treatment of subjects in the lowest third. However, even in the lowest third of the risk spectrum the NNT is 63, a not unimpressive result for a relatively short period of treatment ( 1.6 years).

Weaknesses of the analyses that were conducted are primarily caused by the reduced power associated with the important but modest overall risk reduction. Despite this fact, the consistency of benefit across all subgroups supports the view that the overall results of the study in terms of a $17 \%$ relative reduction for the primary end point can be applied to a wide range of subjects with coronary disease who suffer from angina pectoris.

Current guidelines place $\beta$ blockers as the first line treatment for the medical management of angina pectoris. This is probably because, in addition to being effective antianginal agents, they also have an impressive record in reducing major coronary events across a wide spectrum of coronary heart disease, although this has never been tested in an appropriately powered trial in stable angina. In contrast, calcium channel blockers and then long acting nitrates, which are added in or substituted in cases of inadequate efficacy or intolerance to $\beta$ blockers, have not been shown to have cardioprotective effects in coronary heart disease. Therefore, it would appear that IONA has two important messages for clinical practice. Firstly, in this group of patients with established coronary heart disease, nicorandil may be added to statins, antiplatelet agents, and ACE inhibitors as a member of a class of treatments that prevent major clinical events. Secondly, the secondary prevention properties established in IONA, combined with the already known antianginal properties of nicorandil, suggests that nicorandil should be considered at an earlier stage in the treatment of angina ahead of calcium channel blockers and nitrates.

\section{ACKNOWLEDGEMENTS}

The IONA study was sponsored by Merck Pharmaceutical, Aventis Pharma, and the Chugai Pharmaceutical Company.

Conflict of interest statement: Members of the various committees received research grants or honoraria in respect of their contribution to this study. The academic members of the Scientific Steering Committee have complete access to the study data and have full rights to publish the results of the study independently of the sponsors.

\section{APPENDIX: IONA STUDY GROUP}

\section{Steering committee}

Voting members

Professor HJ Dargie (Chairman, Glasgow Western Infirmary, Glasgow), Professor I Ford (Glasgow University, Glasgow), Professor K M Fox (Royal Brompton Hospital, London), Professor W S Hillis (Glasgow University, Glasgow).

\section{Sponsor representatives (non-voting)}

Dr M Morris (Merck Pharmaceuticals Ltd), Dr M Ford (Aventis Pharma Ltd).

\section{Critical events committee}

Professor WS Hillis (Glasgow University, Glasgow), Professor JJV McMurray (Glasgow University, Glasgow), Dr AL Clark (University of Hull, Kingston upon Hull).

\section{Data and safety monitoring committee}

Professor J Hampton (Chairman, University Hospital, Nottingham), Dr A Skene (Nottingham Clinical Research Ltd), Dr J Birkhead (Northampton General Hospital, Northampton).

\section{Statistical and data centre}

Robertson Centre for Biostatistics, University of Glasgow, Glasgow: Professor I Ford (Director), Dr A McMahon (Study Statistician), Ms A Trainer, Ms H Christie (Database Managers), Ms A Nears (Nurse/coder), Ms C Ferrell (Study Administrator), Dr B Shaw (Clinical Co-ordinator).

\section{Study monitoring}

Ingenix Pharmaceutical Services: Ms V Diment (Project Manager) 
Investigators

See IONA Study Group. ${ }^{9}$

\section{REFERENCES}

1 Tunstall-Pedoe H. Angina pectoris: epidemiology and risk factors. Eur Heart J 1985;6:1-5.

2 Gandhi MM. Clinical epidemiology of coronary heart disease in the UK. Br J Hosp Med 1997; 58:23-7.

3 Simon S, Niamh M, Andrew W, et al. The current cost of angina pectoris to the National Health Service in the UK. Heart 2003;89:848-53.

4 Antiplatelet Trialists' Collaboration. Collaborative overview of randomised trials of antiplatelet therapy. Prevention of death, myocardial infarction, and stroke by prolonged antiplatelet therapy in various categories of patients. BMJ 1994;308:81-106.

5 Yusuf S, Sleight $\mathrm{P}$, Pogue J, et al. Effects of an angiotensin-converting-enzyme inhibitor, Ramipril, on cardiovascular events in high-risk patients. N Engl J Med 2000;342: 145-53.

6 Scandinavian Simvastatin Survival Study Group. Randomised trial of cholesterol lowering in 4444 patients with coronary heart disease: the Scandinavian simvastatin survival study (4S). Lancet 1994;344:1383-9.

7 LIPID Study Group. Prevention of cardiovascular events and death with pravastatin in patients with coronary heart disease and a broad range of initial cholesterol levels. N Engl J Med 1998:339:1349-57.

8 Rubins HB, Sander JR, Collins D, et al. Gemfibrozil for the secondary prevention of coronary heart disease in men with low levels of high density lipoprotein cholesterol. N Engl J Med 1999:341:410-8.

9 IONA Study Group. Effect of nicorandil on coronary events in patients with stable angina: the impact of nicorandil in angina (IONA) randomised trial. Lancet 2002;359:1269-75.
10 Chibana T, Nagamine F, Sunagawa R, et al. Comparison of acute hemodynamic and coronary vasodilating effects between nicorandil and glyceryl trinitrate. Arzneim-Forsh/Drug Res 1991;41:6:591-4.

11 Treese N, Erbel R, Meyer J. Acute hemodynamic effects of nicorandil in coronary artery disease. J Cardiovas Pharmacol 1992;20:S3:52-6.

12 Taira N. Similarity and dissimilarity in the mode and mechanism of action between nicorandil and classical nitrates: an overview. J Cardiovas Pharmacol 1987; 10:S1-9.

13 Taira N. Nicorandil as a hybrid between nitrates and potassium channel activators. J Cardiol 1989:63:18-24J.

14 Doring G. Antianginal and anti-ischemic efficacy of nicorandil in comparison with isosorbide-5-mononitrate and isosorbide dinitrate: results from two multicenter, double-blind, randomized studies with stable coronary heart disease patients. J Cardiovasc Pharm 1992;20:S74-81.

15 Di Somma S, Liguori V, Verdecchia P, et al. A double-blind comparison of nicorandil and metoprolol in patients with effort stable angina. Cardiovasc Drugs Ther 1993;7:119-23.

16 Swan Study Group. Comparison of the antiischaemic and antianginal effect of nicorandil and amlodipine in patients with symptomatic stable angina pectoris: the SWAN study. J Clin Basic Cardiol 1999;2:213-7.

17 Patel DJ, Purcell J, Fox KM. Cardioprotection by opening of the KATP channel in unstable angina. Eur Heart J 1999;20:51-7.

18 Tomai F, Crea F, Gaspardone A, et al. Mechanisms of cardiac pain during coronary angioplasty. J Am Coll Cardiol 1993;22:1892-6.

19 Yellon DF, Alkulaifi AM, Pugsley WB. Preconditioning the human myocardium. Lancet 1993;342:276-7.

20 Jenkins DP, Pugsley WB, Alkhulaifi AM, et al. Ischaemic preconditioning reduces troponin $T$ release in patients undergoing coronary bypass surgery. Heart 1997;77:314-8.

21 IONA Study Group. Impact of nicorandil in angina (IONA): design, methodology and management. Heart 2001;85:E9.

\section{IMAGES IN CARDIOLOGY}

\section{Giant left main coronary artery aneurysm with mitral regurgitation}

$\mathrm{A}$ 32 year old woman presented with acute myocardial infarction and left ventricular failure leading to acute mitral regurgitation. A coronary angiogram revealed a proximal stenosis of the left anterior descending artery (LAD), occlusion of the circumflex artery, and an aneurysm of the left main coronary artery, measuring $24 \times 16 \mathrm{~mm}$ (panel A). A transoesophageal echocardiogram showed grade III mitral valve regurgitation secondary to annular dilatation and lateral wall dysfunction. The patient underwent surgery three weeks later. During the operation, the left main stem aneurysm containing multiple thrombi was over-sewn both proximally and distally. The LAD was grafted with the left internal thoracic artery, and intermediate and obtuse marginal arteries were grafted sequentially with a segment of the long saphenous vein. Failure in weaning off from cardiopulmonary bypass, because of the hypoperfusion within the LAD distribution, led to an additional vein graft to the LAD. The mitral valve was repaired using an annuloplasty band. The patient remains clinically asymptomatic; follow up computed tomographic coronary angiography six months later revealed an occluded left main stem and the aneurysm, and patent saphenous vein grafts to the LAD (panel B and C). As there are reports that such patients
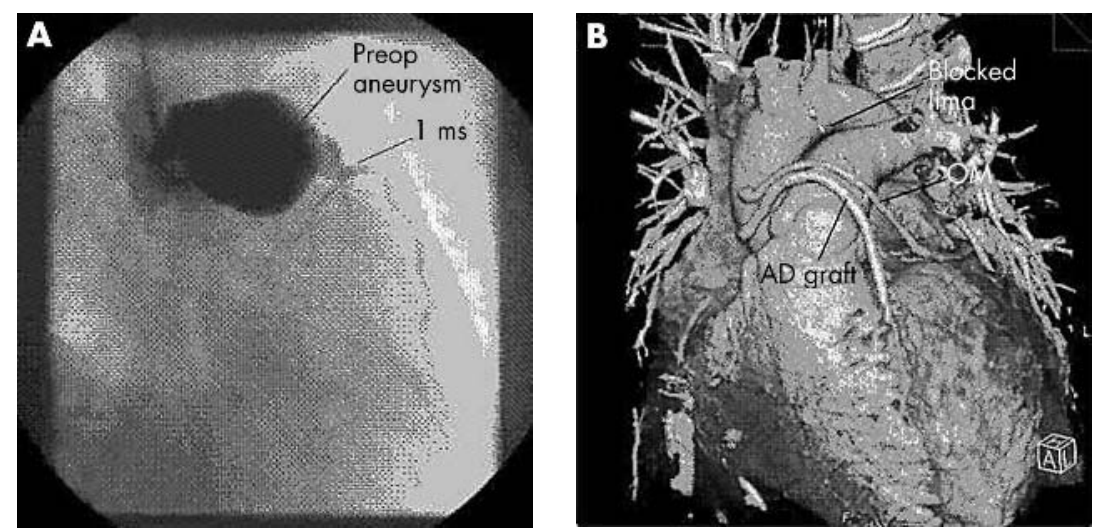

may also develop extracardiac aneurysms, she underwent a magnetic resonance imaging scan of head, thorax, and abdomen, which did not show any pathology.

Aneurysms of the left main coronary artery occur in $0.1 \%$ of adults. Only a few descriptions exist of a surgically treated aneurysm of the left main coronary artery. In this case, a direct approach was undertaken to close the aneurysm, and this was combined with mitral valve repair for ischaemic mitral regurgitation.

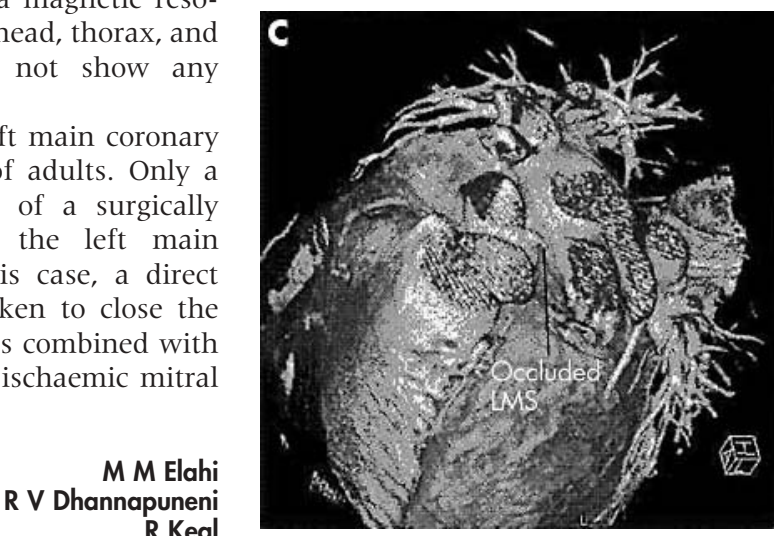

